\title{
Alliuocide G, a new flavonoid with potent $\alpha$-amylase inhibitory activity from Allium cepa $\mathrm{L}$.
}

\author{
Gamal A. Mohamed \\ Department of Pharmacognosy, Faculty of Pharmacy, Al-Azhar University, Assiut Branch \\ 71524, Assiut, Egypt \\ E-mail:gamals2001@yahoo.com
}

\begin{abstract}
Chemical investigation of the ethyl acetate fraction of the dried outer scales of Allium cepa L. afforded one new flavonoid named alliuocide $G$, together with four known compounds. Their structures were unambiguously established on the bases of NMR spectroscopy $\left({ }^{1} \mathrm{H},{ }^{13} \mathrm{C}\right.$, DEPT, and $\mathrm{HMBC}$ ) and mass spectrometry. The isolated compounds showed potent $\alpha$-amylase inhibitory activities, in addition the new compound had antioxidant activity.
\end{abstract}

Keywords: Allium cepa L. alliuocide G, antioxidant and $\alpha$-amylase inhibitory activities

\section{Introduction}

High dietary intakes of fruits and vegetables have been reported to correlate with a low risk of degenerative diseases from epidemiological evidence. The protective effects have been attributed partly to the various antioxidative compounds present in fruits and vegetables. The most abundant types of antioxidative compounds in human diet are flavonoids ${ }^{1}$. Previous phytochemical studies of the A. Cepa L. (family: Liliaceae) (white and red onion) have resulted the isolation of flavonoids ${ }^{2-6}$, especially high levels of quercetin and its derivatives ${ }^{2}$, anthocyanins $^{7,8}$, thiosulfinates, sulfides, sulfoxides ${ }^{3}$ and peptides ${ }^{9}$. This study undertook the isolation and structure elucidation of one new flavonoid named alliuocide $G$, together with four known compounds. The isolated compounds were tested for their $\alpha$-amylase inhibitory activities, in addition the new compound was evaluated for its antioxidant activity using DPPH assay.

\section{Results and Discussion}

The methanolic extract of the air-dried brownish scales of onions was concentrated to dryness and successively partitioned between $n$-hexane, ethyl acetate, $n$-butanol and water. The ethyl 
acetate fraction afforded one new flavonoid named alliuocide $G(2)$ and four known compounds (1, 3-5).

Compound 2 was obtained as brownish amorphous powder (18.6 mg). It had a molecular weight of 426 as derived from the ESI-MS measurement which in conjunction with ${ }^{1} \mathrm{H},{ }^{13} \mathrm{C}$ and DEPT NMR spectral data suggested the molecular formula $\mathrm{C}_{21} \mathrm{H}_{14} \mathrm{O}_{10}$. The UV spectrum showed absorbance at $\lambda_{\max }(\mathrm{MeOH}) 290 \mathrm{~nm}$ indicated the lack of conjugation between $\mathrm{C}-2$ and $\mathrm{C}-3$ of the quercetin moiety ${ }^{1}$. IR spectrum showed absorption bands at $\gamma_{\max }(\mathrm{KBr}) \mathrm{cm}^{-1} ; 3455(\mathrm{OH})$, $1658(\mathrm{C}=\mathrm{O})$ and $1556(\mathrm{C}=\mathrm{C})$. The ${ }^{1} \mathrm{H}$ NMR spectrum showed the presence of two protons at $\delta_{\mathrm{H}}$ 5.94 (brs, H-6/8), one ortho coupled at $\delta_{\mathrm{H}} 6.67$ (d, $\left.J=8.5 \mathrm{~Hz}, \mathrm{H}-5^{\prime}\right)$, one ortho and meta coupled at $\delta_{\mathrm{H}} 6.90\left(\mathrm{dd}, J=8.5,1.8 \mathrm{~Hz}, \mathrm{H}-6^{\prime}\right)$ and one meta downfield coupled proton at $\delta_{\mathrm{H}} 7.10(\mathrm{~d}, J=$ $\left.1.8 \mathrm{~Hz}, \mathrm{H}-2^{\prime}\right)$ indicated the presence of quercetin moiety. In addition, three proton signals at $\delta_{\mathrm{H}}$ $7.15\left(\mathrm{~d}, J=8.5 \mathrm{~Hz}, \mathrm{H}-5^{\prime \prime}\right), 7.46\left(\mathrm{~d}, J=1.9 \mathrm{~Hz}, \mathrm{H}-2^{\prime \prime}\right)$ and $7.58\left(\mathrm{dd}, J=8.5,1.9 \mathrm{~Hz}, \mathrm{H}-6^{\prime \prime}\right)$ were observed, attributed to a tri-substituted benzene moiety. The ${ }^{13} \mathrm{C}$ spectrum showed resonances for twenty one carbon signals, one downfield quaternary signal at $\delta_{\mathrm{C}} 187.1(\mathrm{C}-4)$, two characteristic quaternary carbons at $\delta_{\mathrm{C}} 100.0(\mathrm{C}-2)$ and 90.8 (C-3). These data indicated that compound 2 consisting of quercetin in addition to a tri-substituted benzene moiety, in which oxidative coupling of both conjugated olefinic linkage (C-2 and C-3) of the quercetin C-ring and the orthodihydroxy group (C-3"' and C-4"') of 1,3,4-trihydroxy benzene ring. The structure assignment was supported by HMBC spectral analysis of cross peaks: the proton signal $\mathrm{H}-2$ " gave cross peaks with carbon signals resonating at $\delta_{\mathrm{C}} 124.4$ (C-6"'), 140.3 (C-3') 144.7 (C-4") and 166.1 $\left(\mathrm{C}-1^{\prime \prime}\right), \mathrm{H}-5^{\prime \prime}$ with C-6" $\left(\delta_{\mathrm{C}} 124.4\right), \mathrm{C}-3^{\prime \prime}\left(\delta_{\mathrm{C}} 140.3\right), \mathrm{C}-4^{\prime \prime}\left(\delta_{\mathrm{C}} 144.7\right), \mathrm{H}-6^{\prime \prime}$ with C-2" $\left(\delta_{\mathrm{C}}\right.$ $117.9), \mathrm{C}-5^{\prime \prime}\left(\delta_{\mathrm{C}} 118.4\right), \mathrm{C}-4^{\prime \prime}\left(\delta_{\mathrm{C}} 144.7\right)$, and C-1" $\left(\delta_{\mathrm{C}} 166.1\right)$. Furthermore, H-6 showed HMBC correlations with C-5 $\left(\delta_{\mathrm{C}} 163.1\right), \mathrm{C}-7\left(\delta_{\mathrm{C}} 166.1\right), \mathrm{C}-8\left(\delta_{\mathrm{C}} 96.3\right)$ and H-8 with C-4 $\left(\delta_{\mathrm{C}}\right.$ $187.1), C-6\left(\delta_{C} 97.3\right), C-9\left(\delta_{C} 159.1\right)$ and $C-10\left(\delta_{C} 100.2\right)$. In addition, the connectivity of ring-D to ring-C at C-2 and C-3 of quercetin moiety was confirmed by the upfield shifts of C-2 $\left(\delta_{\mathrm{C}}\right.$ $100.2)$ and $\mathrm{C}-3\left(\delta_{\mathrm{C}} 90.8\right)$. To the best of our knowledge compound $\mathbf{2}$ is a new natural product and named alliuocide $\mathrm{G}$. The known compounds were identified by analysis of the spectroscopic data (NMR and MS) and comparison of their data with those reported in the literature to be: 2-(3,4dihydroxybenzoyl)-2,4,6-trihydroxy-3 (2H)-benzofuranone $(\mathbf{1})^{1}, 1,3,11 \alpha$-trihydroxy-9-(3,5,7trihydroxy-4H-1-benzopyran-7-on-2-yl)-5 $\alpha$-(3,4-dihydroxyphenyl)-5,6,11-hexahydro-5,6,11-

trioxanaphthacene-12-one (quercetin dimer) $(3)^{1,5}$, luteolin-7-O- $\beta$-D-glucopyranoside $(4)^{10,11}$ and [1,3,11 $\alpha$-trihydroxy-9-(3,5,7-trihydroxy-4H-1-benzopyran-7-on-2-yl)-5 $\alpha$-(3,4-dihydroxyphenyl)5,6,11-hexahydro-5,6,11-trioxana-phthacene-12-one]-4'- $O$ - $\beta$-D-gluco-pyra-noside $\quad\left(4^{\prime}-O\right.$ - $\beta$-Dglucopyranoside of quercetin dimer) $(5)^{1,5}$. This is the first report of compound (4) in the plant, while compounds $(\mathbf{1}, \mathbf{3}$ and $\mathbf{5})$ were previously isolated from the same plant ${ }^{1,5}$. 
Table 1. ${ }^{1} \mathrm{H}$ and ${ }^{13} \mathrm{C}$ NMR data of compound 2 (DMSO- $d_{6}, 500,125 \mathrm{MHz}$ )

\begin{tabular}{|c|c|c|c|}
\hline Pos. & $\delta_{\mathrm{H}}(J \mathrm{~Hz}, \mathrm{~m})$ & $\delta_{\mathrm{C}}(\mathrm{m})$ & HMBC \\
\hline 2 & & $100.0 \mathrm{~s}$ & \\
\hline 3 & & $90.8 \mathrm{~s}$ & \\
\hline 4 & & $187.1 \mathrm{~s}$ & \\
\hline 5 & & $163.1 \mathrm{~s}$ & \\
\hline 6 & $5.94,1 \mathrm{H}, \mathrm{brs}$ & $97.3 \mathrm{~d}$ & $5,7,8,10$ \\
\hline 7 & & $166.6 \mathrm{~s}$ & \\
\hline 8 & $5.94,1 \mathrm{H}, \mathrm{brs}$ & $96.3 \mathrm{~d}$ & $4,6,9,10$ \\
\hline 9 & & $159.1 \mathrm{~s}$ & \\
\hline 10 & & $100.2 \mathrm{~s}$ & \\
\hline 1 & & $124.2 \mathrm{~s}$ & \\
\hline 2 & $7.10,1 \mathrm{H}, \mathrm{d}, J=1.8 \mathrm{~Hz}$ & $115.7 \mathrm{~d}$ & $2,3^{\prime}, 4,6$ \\
\hline 3 & & $144.4 \mathrm{~s}$ & \\
\hline 4 & & $147.0 \mathrm{~s}$ & \\
\hline 5 & $6.67,1 \mathrm{H}, \mathrm{d}, J=8.5 \mathrm{~Hz}$ & $114.8 \mathrm{~d}$ & $1 ', 3,4$ \\
\hline 6 & $6.90,1 \mathrm{H}, \mathrm{dd}, J=8.5,1.8 \mathrm{~Hz}$ & $119.3 \mathrm{~d}$ & $2,5,4$ \\
\hline $1^{\prime \prime}$ & & $166.6 \mathrm{~s}$ & \\
\hline 2 & $7.46,1 \mathrm{H}, \mathrm{d}, J=1.9 \mathrm{~Hz}$ & $117.9 \mathrm{~d}$ & $1^{\prime \prime}, 3^{\prime}, 4^{\prime}, 6^{\prime}$ \\
\hline $3 "$ & & $140.3 \mathrm{~s}$ & \\
\hline $4 "$ & & $144.7 \mathrm{~s}$ & \\
\hline $5^{\prime \prime}$ & $7.15,1 \mathrm{H}, \mathrm{d}, J=8.5 \mathrm{~Hz}$ & $118.4 \mathrm{~d}$ & $3^{\prime \prime}, 4^{\prime \prime}, 6^{\prime \prime}$ \\
\hline 6 & $7.58,1 \mathrm{H}, \mathrm{dd}, J=8.5,1.9 \mathrm{~Hz}$ & $124.4 \mathrm{~d}$ & $1^{\prime}, 2 “, 4^{\prime}, 5^{\prime}$ \\
\hline $\mathrm{OH}$ & $10.85,9.18,8.89$, each $1 \mathrm{H}, \mathrm{s}$ & & \\
\hline
\end{tabular}

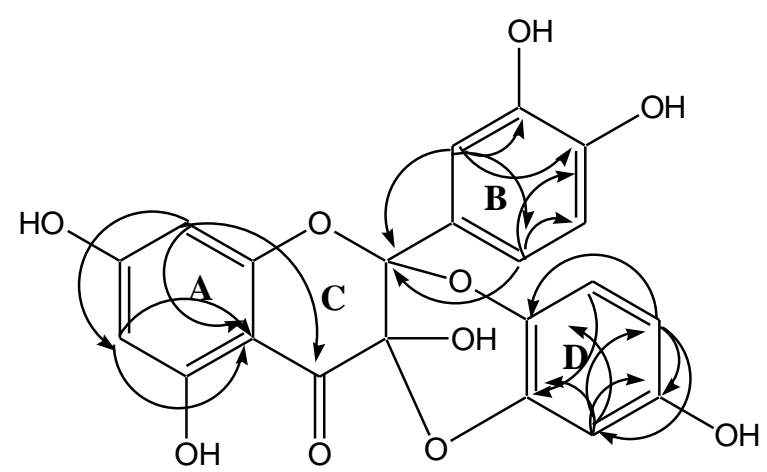

Figure 1. Some important HBMC correlations of compound 2. 

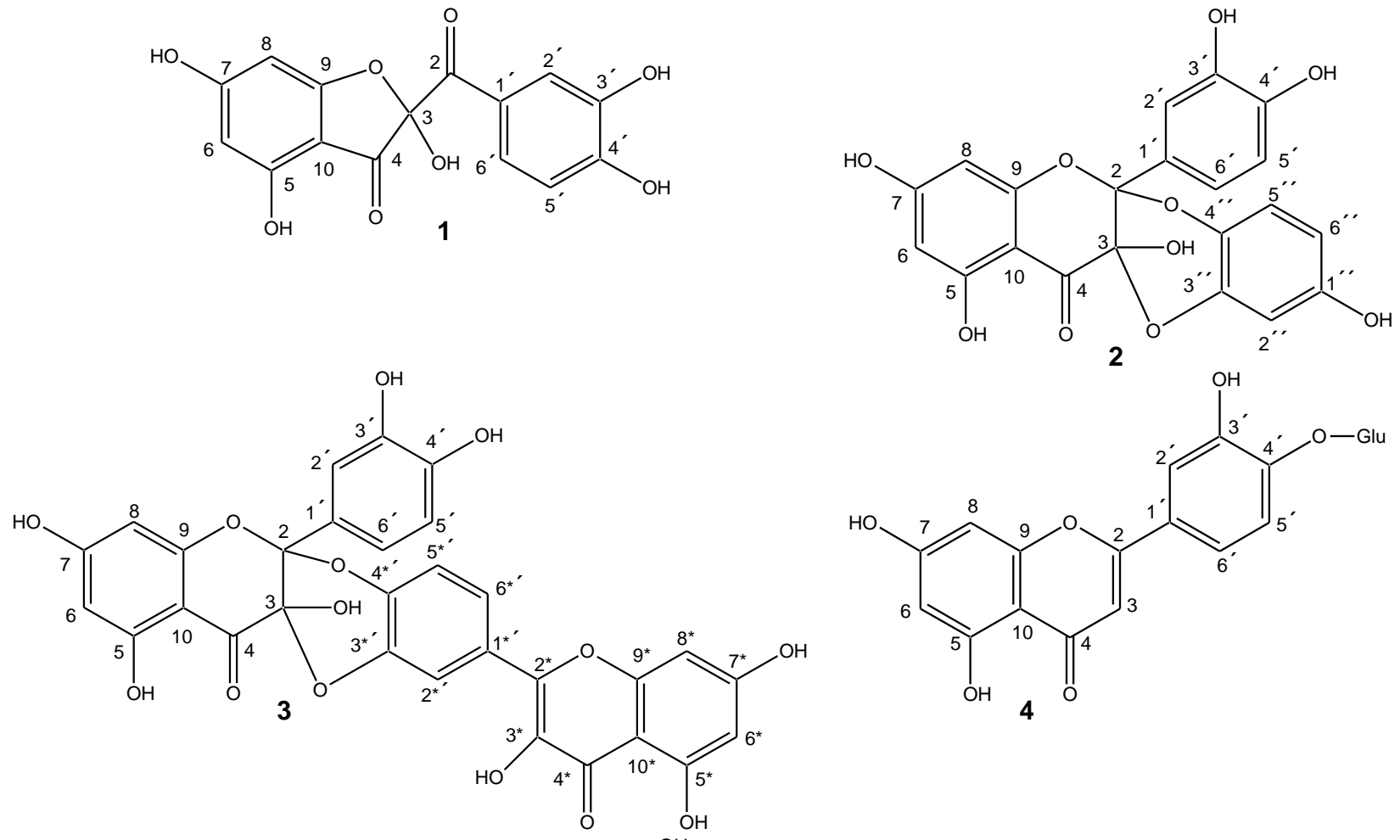<smiles>COc1ccc(C23Oc4ccc(-c5oc6cc(O)cc(O)c6c(=O)c5O)cc4OC2(O)C(=O)c2c(O)cc(O)cc2O3)cc1O</smiles>

Figure 2. Chemical structures of isolated compounds 1-5.

\section{Experimental Section}

General Procedures. Melting points were carried out in Electrothermal 9100 Digital Melting Point (England, Ltd). The UV spectra were carried out in methanol (Merck) using a PerkinElmer Lambda 25 UV/VIS spectrophotometer. IR was measured on Schimadzu Infrared-400 spectrophotometer (Japan). Optical rotation was recorded on a Perkin-Elmer Model 341 LC Polarimeter. $\alpha$-Amylase inhibitory activities were measured in Tecan Genios microplate reader 
(GmbH, Germany). 1D and 2D NMR experiments were performed on Bruker Unity 500, 125 $\mathrm{MHz}$ spectrometer. ESI-MS spectra were obtained with a Thermofinnigan LCQ DECA mass spectrometer coupled to an Agilent 1100 HPLC system equipped with a photodiode array detector. Column chromatographic separation were performed on silica gel 60 (0.04-0.063 mm), RP-18 (0.04-0.063 mm Merck), and Sephadex LH-20 (0.25-0.1 mm Merck). TLC analyses were carried out on aluminum sheets precoated with silica gel $60 \mathrm{~F}_{254}$ (Merck, Darmstadt, Germany). The solvent systems used for TLC analyses were $\mathrm{CH}_{2} \mathrm{Cl}_{2}-\mathrm{MeOH}$ (95:5, solvent system I), $\mathrm{CH}_{2} \mathrm{Cl}_{2}-\mathrm{MeOH}$ (9:1, solvent system II) and $\mathrm{CH}_{2} \mathrm{Cl}_{2}-\mathrm{MeOH}$ (85:15, solvent system III). All solvents were distilled prior to use. Spectral grade solvents were utilized for chromatographic analysis.

\section{Extraction and isolation}

White onions were purchased at the Faculty of Agriculture, Al-Azhar University, Assiut, Egypt. The air-dried brownish scales of onions (400 g) was extracted several times with methanol ( $2 \mathrm{~L} \times$ 5) and evaporated to yield $36 \mathrm{~g}$. The latter was suspended in distilled water $(100 \mathrm{ml})$ then partitioned between $n$-hexane $(500 \mathrm{~mL} \times 4)$, ethyl acetate $(500 \mathrm{~mL} \times 4)$ and $n$-butanol $(500 \mathrm{~mL} \times$ $3)$, successively. Each fraction was concentrated under reduced pressure to give $n$-hexane fraction (4.6 g), ethyl acetate fraction (8.6 g), $n$-butanol fraction (10.3 g) and aqueous (12.2 g) residue. The ethyl acetate fraction $(8.6 \mathrm{~g})$ was subjected to vacuum liquid chromatography (VLC) using $\mathrm{CHCl}_{3}-\mathrm{MeOH}$ gradients to afford 5 fractions. Fraction II (0.96 g) was chromatographed over silica gel column using $\mathrm{CHCl}_{3}-\mathrm{MeOH}$ gradients to get compound 1 (32 $\mathrm{mg}$ ). Fraction III (2.3 g) was chromatographed on Sephadex LH-20 eluted with MeOH to yield subfractions A-C. Subfraction A (0.38 g) was subjected to silica gel column using $\mathrm{CHCl}_{3}-\mathrm{MeOH}$ gradients to afford compound $3(26 \mathrm{mg})$. Subfraction C (0.71 g) was subjected to RP-18 column using $\mathrm{MeOH}-\mathrm{H}_{2} \mathrm{O}$ gradient to give compound 2 (18 mg). Fraction $\mathrm{V}(3.7 \mathrm{~g})$ was chromatographed over silica gel column using $\mathrm{CHCl}_{3}-\mathrm{MeOH}$ gradients to obtain compound 4 (13 mg), and 5 (44 mg). Further purification of compounds 4 and 5 were accomplished by RP-18 column eluted with $\mathrm{MeOH}-\mathrm{H}_{2} \mathrm{O}$ gradients.

2-(3,4-Dihydroxybenzoyl)-2,4,6-trihydroxy-3 (2H)-benzofuranone (1). ${ }^{1}$ Brown amorphous solid; $\mathrm{R}_{f}=0.53$ (solvent system I); m.p. 213-214 ${ }^{\circ} \mathrm{C}$, UV $\lambda_{\max }(\mathrm{MeOH}): 292 \mathrm{~nm}$. (-) ESI-MS $\mathrm{m} / z$ (rel. int.\%): $317.2[\mathrm{M}-\mathrm{H}]^{-}$(100). ${ }^{1} \mathrm{H}$ NMR data (DMSO-d6, $\left.500 \mathrm{MHz}\right): \delta_{\mathrm{H}} 5.89$ (1H, brs, H-6), 5.94 (1H, brs, H-8), 6.79 (1H, d, $\left.J=8.3 \mathrm{~Hz}, \mathrm{H}^{-} 5^{\prime}\right), 7.54$ (1H, dd, $\left.J=8.3,1.9 \mathrm{~Hz}, \mathrm{H}-6^{\prime}\right), 7.55$ $\left(1 \mathrm{H}, \mathrm{d}, J=1.9 \mathrm{~Hz}, \mathrm{H}-2^{\prime}\right), 9.46$ and 8.69 (each $1 \mathrm{H}, \mathrm{s}, \mathrm{OH}$-groups).

1,3,9,11 $\alpha$-Tetrahydroxy-5 $\alpha$-(3,4-dihydroxyphenyl)-5,11-dihydro-5,6,11-trioxa naphthacene12-one (Alliuocide G) (2). Brownish amorphous solid; $\mathrm{R}_{f}=0.71$ (solvent system II); $[\alpha]_{\mathrm{D}}+$ $71.4^{\circ}$ ( $c$ 0.5, MeOH). m.p. 292-293 ${ }^{\circ} \mathrm{C}$, UV $\lambda_{\max }(\mathrm{MeOH}): 290 \mathrm{~nm}$. (+) ESI-MS $\mathrm{m} / z$ (rel. int.\%): $427.2[\mathrm{M}+\mathrm{H}]^{+}(100),(-)$ ESI-MS m/z (rel. int.\%): $425.1[\mathrm{M}-\mathrm{H}] \mathrm{p}(86) . \mathrm{IR} \gamma_{\max }(\mathrm{KBr}) \mathrm{cm}^{-1}: 3455$, 2986, 1658, 1556, 1057, 583. ${ }^{1} \mathrm{H}$ NMR data (DMSO- $\left.d_{6}, 500 \mathrm{MHz}\right): \delta_{\mathrm{H}} 5.94$ (2H, brs, H-6/8), $6.67\left(1 \mathrm{H}, \mathrm{d}, J=8.5 \mathrm{~Hz}, \mathrm{H}-5^{\prime}\right), 6.90\left(1 \mathrm{H}, \mathrm{dd}, J=8.5,1.8 \mathrm{~Hz}, \mathrm{H}-6^{\prime}\right), 7.10(1 \mathrm{H}, \mathrm{d}, J=1.8 \mathrm{~Hz}, \mathrm{H}-$ $\left.2^{\prime}\right), 7.15$ (1H, d, $\left.J=8.5 \mathrm{~Hz}, \mathrm{H}-5^{\prime \prime}\right), 7.46$ (1H, d, $\left.J=1.9 \mathrm{~Hz}, \mathrm{H}-2^{\prime \prime}\right), 7.58$ (1H, dd, $J=8.5,1.9$ 
$\mathrm{Hz}, \mathrm{H}-6^{\prime \prime}$ ), 8.89, 9.18 and 10.85 (each $1 \mathrm{H}, \mathrm{s}, \mathrm{OH}$-groups). ${ }^{13} \mathrm{C}$ NMR (DMSO- $d_{6}, 125 \mathrm{MHz}$ ): $\delta_{\mathrm{C}}$ 90.8 (C-3, s), 96.3 (C-8, d), 97.3 (C-6, d), 100.0 (C-2, s), 100.2 (C-10, s), 114.8 (C-5' , d), 115.7 (C-2', d), 117.9 (C-2”', d), 118.4 (C-5'”, d), 119.3 (C-6', d), 124.2 (C-1', s), 124.4 (C-6”', d), $140.3\left(\mathrm{C}-3^{\prime \prime}, \mathrm{s}\right), 144.4$ (C-3', s), 144.7 (C-4'”, s), 147.0 (C-4', d), 159.1 (C-9, s), 163.1 (C-5, s), $166.6\left(\mathrm{C}-7,1^{\prime \prime}, \mathrm{s}\right), 187.1(\mathrm{C}-4, \mathrm{~s})$.

\section{1,3,11 $\alpha$-Trihydroxy-9-(3,5,7-trihydroxy-4H-1-benzopyran-7-on-2-yl)-5 $\alpha$-(3,4-dihydroxy-} phenyl)-5,6,11-hexahydro-5,6,11-trioxanaphthacene-12-one (Quercetin dimer) (3). 1,5 Yellow amorphous solid; $\mathrm{R}_{f}=0.64$ (solvent system II); m.p. $267-268{ }^{\circ} \mathrm{C}$, UV $\lambda_{\max }(\mathrm{MeOH}): 254$, 272, 300, and $370 \mathrm{~nm}$. (-) ESI-MS $m / z$ (rel. int.\%): 601.1 [M-H] ${ }^{-}(100) .{ }^{1} \mathrm{H}$ NMR data (DMSO- $d_{6}$, $500 \mathrm{MHz}): \delta_{\mathrm{H}} 5.97\left(2 \mathrm{H}\right.$, brs, H-6/8), $6.20\left(1 \mathrm{H}, \mathrm{d}, J=2.8 \mathrm{~Hz}, \mathrm{H}-6^{*}\right), 6.48(1 \mathrm{H}, \mathrm{d}, J=2.7 \mathrm{~Hz}, \mathrm{H}-$ $\left.8^{*}\right), 6.71\left(1 \mathrm{H}, \mathrm{d}, J=8.5 \mathrm{~Hz}, \mathrm{H}-5^{\prime}\right), 6.95\left(1 \mathrm{H}, \mathrm{dd}, J=8.5,2.2 \mathrm{~Hz}, \mathrm{H}-6^{\prime}\right), 7.17(1 \mathrm{H}, \mathrm{d}, J=2.2 \mathrm{~Hz}$, H-2' $), 7.26\left(1 \mathrm{H}, \mathrm{d}, J=8.5 \mathrm{~Hz}, \mathrm{H}-5^{* \prime}\right), 7.84\left(1 \mathrm{H}, \mathrm{dd}, J=8.5,2.2 \mathrm{~Hz}, \mathrm{H}-6^{* \prime}\right), 7.91(1 \mathrm{H}, \mathrm{d}, J=2.2$ $\mathrm{Hz}, \mathrm{H}-2^{* \prime}$ ), 10.99, 9.21 and 8.97 (each $1 \mathrm{H}, \mathrm{s}, \mathrm{OH}$-groups). ${ }^{13} \mathrm{C}$ NMR (DMSO- $d_{6}, 125 \mathrm{MHz}$ ): $\delta_{\mathrm{C}}$ 91.4 (C-3, s), 93.7 (C-8*, d), 96.4 (C-8, d), 97.3 (C-6, d), 98.4 (C-6*, d), 99.8 (C-2, s), 100.2 (C10, s), 103.3 (C-10*, s), 114.9 (C-5', d), 115.7 (C-2', d), 116.4 (C-5 $\left.{ }^{* \prime}, \mathrm{d}\right), 117.6$ (C-2 $\left.{ }^{* \prime}, \mathrm{d}\right), 119.3$

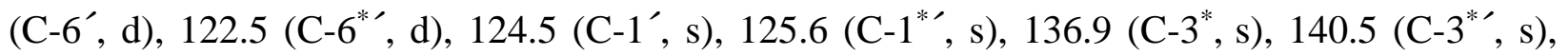
$142.5\left(\mathrm{C}-4^{* \prime}, \mathrm{s}\right), 144.8\left(\mathrm{C}-2^{*}, \mathrm{~s}\right), 145.2\left(\mathrm{C}-3^{\prime}, \mathrm{s}\right), 147.1\left(\mathrm{C}-4^{\prime}, \mathrm{s}\right), 156.4\left(\mathrm{C}-9^{*}, \mathrm{~s}\right), 159.2(\mathrm{C}-9, \mathrm{~s})$, $160.8\left(\mathrm{C}-5^{*}, \mathrm{~s}\right), 163.1$ (C-5, s), 164.3 (C-7*, s), 168.1 (C-7, s), 176.2 (C-4*, s), 187.3 (C-4, s).

Luteolin-7- $\boldsymbol{O}$ - $\boldsymbol{\beta}$-D-glucopyranoside (4). ${ }^{10,11}$ Yellow amorphous solid; $\mathrm{R}_{\boldsymbol{f}}=0.86$ (solvent system III); m.p. 244-245 ${ }^{\circ} \mathrm{C}$, UV $\lambda_{\max }(\mathrm{MeOH}): 266$ and 344 nm. +NaOMe: 273, 300 sh, 389; $+\mathrm{AlCl}_{3}$ : 283, 298 sh, 329, 425; +AlCl $/ \mathrm{HCl}: 281,294$ sh, 358, 384; +NaOAc: 270, 276, 367 sh, 394; $+\mathrm{NaOAc} / \mathrm{H}_{3} \mathrm{BO}_{3}:$ 270, 368. (+) ESI-MS $\mathrm{m} / \mathrm{z}$ (rel. int.\%): 449.1 [M+H] ${ }^{+}(100), 286.9[\mathrm{M}-162$ (glucose) $]^{+}(100),(-)$ ESI-MS $m / z$ (rel. int.\%): $447.3[\mathrm{M}-\mathrm{H}]^{-}(100) .{ }^{1} \mathrm{H}$ NMR data (DMSO- $d_{6}, 500$ $\mathrm{MHz}): \delta_{\mathrm{H}} 4.94\left(1 \mathrm{H}, \mathrm{d}, J=7.1 \mathrm{~Hz}, \mathrm{H}-1^{\prime \prime}\right), 3.44-5.12$ (m, other sugar protons), 6.51 (1H, brs, H6), 6.56 (1H, brs, H-8), 6.98 (1H, s, H-3), 7.41 (1H, d, $\left.J=8.8 \mathrm{~Hz}, \mathrm{H}-5^{\prime}\right), 7.91$ (1H, dd, $J=8.8$, $\left.2.2 \mathrm{~Hz}, \mathrm{H}-6^{\prime}\right), 8.00\left(1 \mathrm{H}, \mathrm{d}, J=2.2 \mathrm{~Hz}, \mathrm{H}-2^{\prime}\right), 12.86(1 \mathrm{H}, \mathrm{s}, 5-\mathrm{OH})$.

\section{[1,3,11 $\alpha$-Trihydroxy-9-(3,5,7-trihydroxy-4H-1-benzopyran-7-on-2-yl)-5 $\alpha$-(3,4-dihydroxy- phenyl)-5,6,11-hexahydro-5,6,11-trioxanaphthacene-12-one $]^{-4}{ }^{\prime}-O$ - $\beta$-D-gluco-pyranoside} (4'-O- $\boldsymbol{\beta}$-D-glucopyranoside of quercetin dimer) (5). ${ }^{1,5}$ Yellow amorphous solid; $\mathrm{R}_{f}=0.76$ (solvent system III); m.p. 301-302 ${ }^{\circ} \mathrm{C}$, UV $\lambda_{\max }(\mathrm{MeOH}): 254,275,303$ and $376 \mathrm{~nm}$. (+) ESI-MS

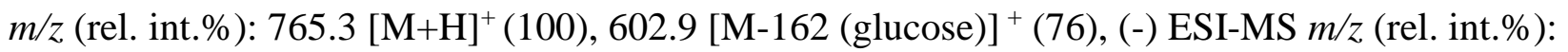
$763.2[\mathrm{M}-\mathrm{H}]^{-}$(100). ${ }^{1} \mathrm{H}$ NMR data (DMSO-d6, $\left.500 \mathrm{MHz}\right): \delta_{\mathrm{H}} 4.92\left(1 \mathrm{H}, \mathrm{d}, J=7.25 \mathrm{~Hz}, \mathrm{H}-1^{\prime \prime}\right)$, 3.45-5.49 (m, other sugar protons), $6.20(1 \mathrm{H}, \mathrm{d}, J=2.2 \mathrm{~Hz}, \mathrm{H}-6), 6.37(1 \mathrm{H}, \mathrm{d}, J=2.2 \mathrm{~Hz}, \mathrm{H}-8)$, $6.40\left(1 \mathrm{H}, \mathrm{d}, J=2.2 \mathrm{~Hz}, \mathrm{H}-6^{*}\right), 6.80\left(1 \mathrm{H}, \mathrm{d}, J=2.2 \mathrm{~Hz}, \mathrm{H}-8^{*}\right), 7.20\left(1 \mathrm{H}, \mathrm{d}, J=8.8 \mathrm{~Hz}, \mathrm{H}-5^{\prime}\right)$, $7.26\left(1 \mathrm{H}, \mathrm{d}, J=8.6 \mathrm{~Hz}, \mathrm{H}-5^{* \prime}\right) 7.42\left(1 \mathrm{H}, \mathrm{d}, J=2.2 \mathrm{~Hz}, \mathrm{H}-2^{\prime}\right), 7.52$ (1H, m, H-6') 7.65 (1H, m, H-6 $\left.{ }^{* \prime}\right), 7.74\left(1 \mathrm{H}, \mathrm{d}, J=2.2 \mathrm{~Hz}, \mathrm{H}-2^{* \prime}\right), 12.58,12.37,10.22,9.69$, and 8.79 (each $1 \mathrm{H}, \mathrm{s}, \mathrm{OH}-$ groups). ${ }^{13} \mathrm{C}$ NMR (DMSO- $\left.d_{6}, 125 \mathrm{MHz}\right): \delta_{\mathrm{C}} 60.5$ (C-6"', t), 69.9 (C-4"', d), 73.2 (C-2”, d), 77.1 (C-5”, d), 77.6 (C-3”, d), 90.9 (C-3, s), 94.3 (C-8* , d), 96.9 (C-8, d), 98.2 (C-6, d), 99.1 (C-6* , d), 100.7 (C-2, s), 101.3 (C-10, s), 104.3 (C-10*, s), 104.0 (C-1'”, d), 115.4 (C-5', d), $116.4\left(\mathrm{C}-2^{\prime}, 5^{* \prime}, \mathrm{d}\right), 117.1$ (C- 2*', d), 120.9 (C-6', d), 124.3 (C-6 $\left.{ }^{* \prime}, \mathrm{d}\right), 129.9$ (C-1', s), 125.6 
$\left(\mathrm{C}-1^{* \prime}, \mathrm{s}\right), 138.1\left(\mathrm{C}-3^{*}, \mathrm{~s}\right), 141.7\left(\mathrm{C}-3^{* \prime}, \mathrm{s}\right), 142.9\left(\mathrm{C}-4^{* \prime}, \mathrm{s}\right), 145.9\left(\mathrm{C}-2^{*}, \mathrm{~s}\right), 146.2\left(\mathrm{C}-3^{\prime}, \mathrm{s}\right)$, 146.9 (C-4', s), 158.2 (C-9*, s), 160.4 (C-9, s), 162.8 (C-5*, s), 165.3 (C-5, s), 165.4 (C-7*, s), $168.9(\mathrm{C}-7, \mathrm{~s}), 177.4\left(\mathrm{C}-4^{*}, \mathrm{~s}\right), 188.6(\mathrm{C}-4, \mathrm{~s})$.

\section{$\boldsymbol{\alpha}$-Amylase inhibitory activity ${ }^{10,12}$}

The method is based on assay of $\alpha$-amylase by EnzCheck ${ }^{\circledR}$ Amylase Assay Kit (E-11954) was purchased from Molecular Probes ( $\mathrm{GmbH}$, Germany). The provided stock solution of DQ starch and $\alpha$-amylase enzyme were diluted with the reaction buffer ( $\mathrm{pH}$ 6.9) according to the reported protocol $^{12}$. To the microplate wells, $50 \mu \mathrm{g} / 10 \mu \mathrm{L}$ of the tested compound in DMSO, $50 \mu \mathrm{L}$ of the diluted enzyme and $40 \mu \mathrm{L}$ of the reaction buffer were added and allowed to stand for $5 \mathrm{~min}$ at room temperature then $100 \mu \mathrm{L}$ of DQ starch was added. The fluorescence intensity of the digestion products from the DQ starch (with or without compounds) was measured using a kinetic assay program in the Tecan Genios microplate reader at $\lambda_{\max } 485 \pm 10 \mathrm{~nm}$ starting from zero min to $60 \mathrm{~min}$ at $10 \mathrm{~min}$ intervals. All determinations were performed in triplicate. The $\alpha$ amylase inhibitory activity of each tested compound was measured in relation to acarbose (Ac) (Kohlpharmam $\mathrm{GmbH}$, Germany) set as $100 \% \alpha$-amylase inhibitory activity. The percentage of $\alpha$-amylase activity and $\alpha$-amylase inhibition was calculated using the following equations:

$\% \alpha$ - amylase activity $=100 \mathrm{x}$

$$
\left(\mathrm{F}_{\mathrm{s}}^{60}-\mathrm{F}_{\mathrm{s}}^{0}\right) /\left(\mathrm{F}_{\mathrm{c}}^{60}-\mathrm{F}_{\mathrm{c}}^{0}\right)
$$

where $\mathrm{F}_{\mathrm{s}}^{60}$ : Fluorescence with the sample at $60 \mathrm{~min}, \mathrm{~F}_{\mathrm{s}}^{0}$ : Fluorescence with the sample at $0 \mathrm{~min}$, $\mathrm{F}_{\mathrm{c}}^{60}$ : Fluorescence of the control at $60 \mathrm{~min}, \mathrm{~F}_{\mathrm{c}}^{0}$ : Fluorescence of the control at $0 \mathrm{~min}$. $\% \alpha$-amylase inhibition $=100-\% \alpha$-amylase activity.

Table 2. $\alpha$-Amylase inhibitory activity of the isolated compounds

\begin{tabular}{cc}
\hline Compounds & Inhibition \% \\
\hline 1 & 88.7 \\
2 & 96.5 \\
3 & 87.7 \\
4 & 82.8 \\
5 & 56.4 \\
\hline
\end{tabular}

All isolated compounds were evaluated for $\alpha$-amylase inhibitory activity as shown in table $\mathbf{2}$, new compound $\mathbf{2}$ showed potent activity compared with acarbose. Apparently, $\alpha$-amylase inhibitory activity increased with the presence of hydroxyl groups at 3`, and 4'-position of the B$\operatorname{ring}^{13}$.

Free radical scavenging activity (DPPH assay) ${ }^{14}$

2,2-Diphenyl-1-picrylhydrazyl (DPPH) and propyl gallate (PG) as reference sample were obtained from Sigma Chemical Co. (Germany). The method was previously described ${ }^{14}$. 
Compound 2 was dissolved in HPLC MeOH to obtain a concentration of $20 \mu \mathrm{m} / \mathrm{mL}$. Then was mixed with DPPH $\left(118 \times 10^{-5}\right)$ and allowed to stand for half an hour for any reaction to occur. The UV absorbance was recorded at $517 \mathrm{~nm}$. The experiment was performed in triplicate and the average absorption was noted. The antioxidant activity was calculated using the following equation;

$$
\text { Antioxidant activity }=100 \times\left(1-\frac{\text { absorbance with compound }}{\text { absorbance of the blank }}\right)
$$

Compound 2 have $66.4 \%$ antioxidant activity according to published structure activity relationship of flavonoids ${ }^{15-18}$ compared with propyl gallate (a known synthetic antioxidant) set as $100 \%$ antioxidant activity.

\section{References}

1. Tram, N. L.; Chiharu, H.; Makoto, S.; Hiromune, A.; Kon, K.; Ryo, Y. J. Agric. Food Chem. 2005, 53, 8183.

2. Yang-Kyun, K.; Chang, Y. L. J. Agric. Food Chem. 1996, 44, 34.

3. Mona, G. Z. MJPS. 2007, 23, 61.

4. Eckhard, W.; Marion, D.; Diego, R.; James, N. R. Z. Naturforsch 2003, 58c, 771.

5. Miyuki, F.; Toshiyuki T.; Ken-ichi, N.; Munekazu, I.; Hironori, T. Heterocycles 2002, 57, 2175.

6. Torgils, F.; Pedersen, A. T.; Øyvind, M.A. Phytochemistry 1998, 47, 281.

7. Torgils, F.; Øyvind, M. A. Phytochemistry 2003, 62, 1217.

8. Torgils, F.; Øyvind, M. A.; Pedersen, A. T.; Ovstedal, D. O.; Raknes, A. J. Food Sci. 1996, $61,703$.

9. Herbert, A. W.; Rudolf, B.; Ingrid, T.; Manuela, L.; Peter, B.; Thomas, S.; Stefan, S.; Roman, C. M. J. Agric. Food Chem. 2005, 53, 3408.

10. Hanaa, M. S.; Mahmoud, H. M.; Salwa, F. F.; Gamal, A. M.; Rainer, E.; Omobuwajo, O. R. M.; Peter, P. Bull. Pharm. Sci., Assiut University 2006, 1, 9.

11. Yuldashev, M. P.; Karimov, A. Chem. Nat. Comp. 2001, 37,413.

12. Molecular Probes website: www.probes.com.

13. Toshiro, M.; Mio, K.; Sachiko, H.; Kiyoshi, M. Biosci. Biotechnol. Biochem. 2002, 66, 689.

14. Kumarasamy, Y.; Byres, M.; Cox, P. J.; Delazar, A.; Jaspars, M.; Nahar, L.; Shoeb, M.; Sarker, S. D. Chem. Nat. Comp. 2004, 40, 122.

15. 15- Pier-Giorgio, P. J. Nat. Prod. 2000, 63, 1035.

16. Stanislaw B.; Wieslaw O. J. Agric. Food Chem. 2001, 49, 2774.

17. Alton, J. D., Jr.; Jose, C. E.; Gloria, C. B.; Kimberly, L. P.; Nikolaus, H. F.; Gary, W. W. J. Nat. Prod. 2000, 63, 327.

18. Cotelle, N.; Bernier, J.; Catteau, J.; Pommery, J.; Wallet, J.; Gaydou, E. M. Free Radic. Biol. Med. 1996, 20, 35. 